\subsection{SEXUALLY TRANSMISSIBLE ENTERIC INFECTIONS IN MEN WHO HAVE SEX WITH MEN: PRELIMINARY FINDINGS FROM A CROSS-SECTIONAL STUDY}

${ }^{1}$ Holly Mitchell ${ }^{*},{ }^{2}$ Gary Whitlock, ${ }^{2}$ Jey Zdravkov, ${ }^{3}$ Jenny Olsson, ${ }^{4}$ Claire Jenkins, ${ }^{5}$ Nicholas Thomson, ${ }^{1}$ Nigel Field, ${ }^{4}$ Gwenda Hughes. ${ }^{1}$ University College London, Centre for Molecular Epidemiology and Translational Research, Institute for Global Health, London, UK; ${ }^{2}$ Chelsea and Westminster Hospital NHS Foundation Trust, 56 Dean Street, London, UK; ${ }^{3}$ Imperial College London, Imperial College School of Medicine, London, UK; ${ }^{4}$ Public Health England, National Infection Service, London, UK; ${ }^{5}$ Wellcome Trust Sanger Institute, Pathogen Genomics, Hinxton, UK

\subsection{6/sextrans-2019-sti.142}

Background Increasing rates of sexually transmissible enteric infections (STEIs) in men who have sex with men (MSM), often associated with antimicrobial resistance, are a growing public health concern. There is a need to better understand the characteristics and burden of STEIs to improve control measures.

Methods We conducted a cross-sectional study at a large London sexual health clinic (SHC) from December 2017 to February 2018. Residual rectal swabs collected from consecutive MSM attending for routine chlamydia/gonorrhoea testing (80\% from asymptomatic screening), were anonymously tested for Shigella, Campylobacter, Salmonella and Escherichia coli by PCR. We generated STEI prevalence estimates and explored factors associated with STEIs using linked socio-demographic, behavioural and clinical data from electronic health records.

Results Of 2,138 specimens tested, overall STEI prevalence was 9.9\% (95\% CI: 8.6\%-11.2\%), ranging from 0.7\% $(95 \%$ CI: $0.4 \%-1.2 \%$ ) for Shigella to $5.0 \%$ (95\% CI: $4.1 \%-6.0 \%$ ) for enteroaggregative E. coli. Salmonella was not detected. MSM with an STEI-positive specimen were more likely to be co-infected with gonorrhoea $(23.7 \%$ vs $16.2 \%, p=0.006)$, to have a previous bacterial STI diagnosis (past year) $(48.3 \%$ vs $37.4 \%, \mathrm{p}<0.002)$, to report an interest in high-risk sexual behaviours' (e.g. Chemsex) $(47.9 \%$ vs $38.7 \%, \mathrm{p}=0.02)$, to report higher partner numbers (past 3 months) (median 6 vs 4, $\mathrm{p}<0.001$ ), and among HIV-negative MSM, to report current use of HIV pre-exposure prophylaxis (PrEP) $(54.7 \%$ vs $35.6 \%, \mathrm{p}<0.001)$. Rectal or gastrointestinal symptoms were reported by $1.9 \%(39 / 2,098)$ of MSM, and this was not associated with overall STEI test result.

Conclusion Nearly one in ten MSM attending a London SHC had a rectal STEI detected. The association with higher-risk sexual behaviour and STIs strengthens the evidence that these pathogens are sexually transmitted. STEIs might be widely underdiagnosed in MSM and sub-clinical infection may support sustained transmission, suggesting the need for well-considered clinical and public health responses.

Disclosure No significant relationships.

\section{4 INCIDENCE OF SEXUAL BEHAVIORS AND RELATIONSHIP TO THE URETHRAL MICROBIOTA AMONG MEN WHO HAVE SEX WITH MEN (MSM) IN SEATTLE}

${ }^{1}$ Laura Chambers*, ${ }^{2}$ James Hughes, ${ }^{3}$ David Fredricks, ${ }^{3}$ Sujatha Srinivasan, ${ }^{4}$ Jennifer Morgan, ${ }^{5}$ Noah Hoffman, ${ }^{4} \mathrm{M}$ Lowens, ${ }^{6}$ Kenneth Tapia, ${ }^{7}$ Sara Glick, ${ }^{1}$ Christine Khosropour, ${ }^{7}$ Matthew Golden, 'Lisa Manhart. 'University of Washington, Epidemiology, Seattle, USA; ${ }^{2}$ University of Washington, Biostatistics, Seattle, USA; ${ }^{3}$ Fred Hutchinson Cancer Research Center, Vaccine and Infectious Disease Division, Seattle, USA; ${ }^{4}$ Public Health - Seattle and King County, HIVISTD Program, Seattle, USA; ${ }^{5}$ University of Washington, Laboratory Medicine, Seattle, USA; ${ }^{6}$ University of Washington, Global Health, Seattle, USA; ${ }^{7}$ University of Washington, Medicine, Seattle, USA

\subsection{6/sextrans-2019-sti.143}

Background Studies suggest that sexual behavior influences the composition of the male urethral microbiota, but this hypothesis has not been tested.

Methods From 12/2014-5/2018, we enrolled MSM with NGU attending an STD clinic into a cohort study. Men attended five in-clinic visits at 3 -week intervals, collected weekly urine specimens at home, and reported daily antibiotics and sex on weekly diaries. We applied broad-range $16 \mathrm{~S}$ rRNA gene PCR with deep sequencing to urine. We estimated incidence of insertive oral sex (IOS) only, condomless insertive anal intercourse (CIAI) only, and IOS with CIAI (IOS+CIAI) after NGU diagnosis using Poisson regression with robust standard errors. We estimated the association between urethral sexual exposures (referent group=none) in seven 3-day time windows before specimen collection and Shannon Index (diversity) and $\log 10$ number of bacterial species (richness) using generalized estimating equations, adjusting for recent antibiotics, age, race/ ethnicity, HIV status, and PrEP use. For each exposure category, we tested whether all seven window coefficients were equal to zero (i.e., no overall association) using a Wald test.

Results Among 92 MSM with NGU, median age was 31 (interquartile range $[\mathrm{IQR}]=28-40) ; 55 \%$ were non-Hispanic white. They contributed 1,095 person-weeks of behavioral data (median $=12$ diaries/man, $\mathrm{IQR}=12-13$ ). Incidence of any sex, IOS only, CIAI only, and IOS+CIAI were $1.07 \quad(95 \%$ confidence interval $[\mathrm{CI}]=0.93-1.24), \quad 0.40 \quad(95 \% \mathrm{CI}=0.32$ $0.49), \quad 0.10 \quad(95 \% \mathrm{CI}=0.07-0.15)$, and $0.40 \quad(95 \% \mathrm{CI}=0.30$ 0.52 ) episodes per person-week, respectively. Among 894 urine specimens (median=10 specimens/man, IQR=8-12), median diversity was $1.33(\mathrm{IQR}=0.76-1.99)$, and median richness was 14 species $(\mathrm{IQR}=9-23)$. Overall, CIAI only $(P<0.01$ in each model) but not IOS only or IOS+CIAI in the prior 21 days was associated with diversity and $\log 10$-richness. Diversity and log10-richness were lower 1-3 days after and higher 16-18 days after CIAI only.

Conclusion Among MSM after NGU, CIAI only in the prior 21 days was independently associated with diversity and richness of the urethral microbiota.

Disclosure No significant relationships. 